\title{
Serious Game for Upper Limb Amputees Rehabilitation
}

\author{
Reidner Santos Cavalcante \\ Faculdade de Engenharia Eletrica \\ Universidade Federal de Uberlandia \\ Uberlandia, MG - Brasil \\ reidnersc@outlook.com
}

\author{
Alexandre Cardoso \\ Faculdade de Engenharia Eletrica \\ Universidade Federal de Uberlandia \\ Uberlandia, MG - Brasil \\ alexandre@ufu.br
}

\author{
Edgard Afonso Lamounier Junior \\ Faculdade de Engenharia Eletrica \\ Universidade Federal de Uberlandia \\ Uberlandia, MG - Brasil \\ lamounier@ufu.br \\ Gerson Mendes de Lima \\ Faculdade de Engenharia Eletrica \\ Universidade Federal de Uberlandia \\ Uberlandia, MG - Brasil \\ gersonlima@ieee.org
}

\author{
Alcimar Soares \\ Faculdade de Engenharia Eletrica \\ Universidade Federal de Uberlandia \\ Uberlandia, MG - Brasil \\ alcimar@ufu.br
}

\begin{abstract}
This work presents a Virtual Reality training environment for upper limb amputees. Based on principles of a serious game, the training environment aims to teach the patient how to control a virtual prosthesis, that lately, will be printed and attached to his forearm. Using a tether with different sensors the patient can interact with the virtual environment. The training protocols were provided by health-care professionals and the interaction technology was developed under their supervision, to ensure high levels of mobility and comfort for the user that are attached to the remaining forearm of the amputee. It was applied a questionnaire that evaluates several points of the game. It was observed that the methods and techniques used for the development of the serious game were shown to be consistent and adequate for the proposed goal.
\end{abstract}

Keywords-Virtual Environment, Training, Amputees, Rehabilitation, Upper limbs

\section{INTRODUCTION}

The goal of a rehabilitation program is to provide the amputee with the skills to perform all possible activities without the use of the prosthesis, to prepare the amputation stump so that it can be protetized and to develop exercises in order to provide functional independence to this user with a equipped limb, making possible the return to the society and its activities of daily living [1]

The earlier the rehabilitation process starts, the greater the potential for success. The longer the delay, the more likely there will be the development of secondary complications such as joint contractures, general debilitation, and a depressed psychological state. The postoperative program can be divided into two phases: (i) the pre-prosthetic phase, which is the period between the surgery and the application of a definitive prosthesis, or until a decision is taken not to use the prosthesis, and (ii) the prosthetic phase, which begins with the delivery of a permanent artificial limb [2].

This work proposes the development of a virtual environment using Virtual Reality as a way of assisting in the rehabilitation process of the patient before obtaining the real prosthesis. In several cases the patient's adaptation process to the real prosthesis is tiring, time consuming, with good chances of motivation loss in the process.

The long time needed for the treatment and the low motivation observed in traditional methods are indicated as reasons for leaving physiotherapeutic treatment, being one of the main causes of therapeutic failure [3]. Therefore, it is important to prepare the amputee to use the prosthesis through an efficient training process.

Virtual Reality is an advanced user interface, having as characteristics the visualization of, and movement in three dimensional environments in real time and the interaction with its elements [4]. With the help of Virtual Reality the system proposed in this work allows the patient to safely experience real-life situations while learning to perform various tasks that will be developed in their daily lives once they acquire the real prosthesis.

\section{MATERIALS AND METHODS}

\section{A. Employed Technologies}

The virtual environment was created using the Unity 3D engine version 5.5.0, while the virtual prosthesis and objects modeling was made inside 3D Studio Max software. The C\# programming language was used to elaborate the application scripts.

For the coding of the application, a Dell Inspiron 5557 notebook was used, with a sixth generation Intel Core i7 processor operating at 2.50 gigahertz, 8 gigabytes of RAM running Windows 10 Home and a dedicated NVIDIA GeForce 930M video card with 4 gigabytes of video memory.

\section{B. Tether, Sensors and the Virtual Environment}

An Ottobock 21A36 = 1 tether model, an equipment consisting of a wire attached to a ring aligned with the center of the user's back was used with a group of sensors. The group of sensors consists of an Arduino Nano with a 10 degrees-offreedom GY-88 Inertial Measurement Unit combining several motion sensors: a MPU6050 (combination of a 3-axis gyroscope and 3-axis accelerometer with digital motion processor) and a HMC5883L (3-axis digital compass from Honeywell manufacturer), which allow the device to identify its position and orientation in the real environment.

Also, a $10 \mathrm{~K} \Omega$ Linear Sliding Potentiometer is connected to the tether cable so that when the individual moves the shoulder, applying a force on the tie rod, the potentiometer cursor slides in the same direction and the value of the displacement is sent for the application and open or close the virtual prosthesis.

The training environment, Fig. 1, consists of five levels that have different objects and difficulties. The degree of difficulty is gradually increased as the user advances the levels. The protocol used in game consists in presenting to the 
user a series of activities in which he must move objects with distinct sizes and shapes from a initial point to a target point defined in the virtual environment. The training protocol to be performed was proposed by a health professional.

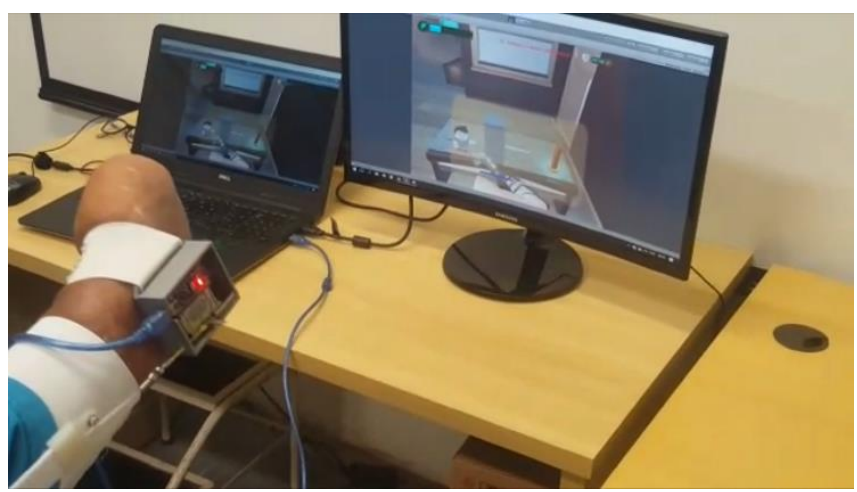

Fig. 1. Amputee using the Virtual Environment. The sensors are attached to the remaining forearm.

\section{Evaluation}

The tests were performed with seven non-amputated users and one amputated user, thus enabling the evaluation of the system based on different points of view, having the amputee's as a priority. The tasks to be performed consisted of moving the object in scene from the point of origin to a highlighted area. At the end of the test a serious game evaluation questionnaire was applied, preceded by na explanation of each question and the specific terms so that the participant was aware of the process. The questionnaire applied included the following key points:

- Usability: evidences the ease of use of the software;

- Functionality: it shows that the set of features meets the explicit and implicit requirements, considering the software purpose: rehabilitation for amputees in order to minimize the time of adaptation to a prosthesis;

- Efficiency: evidences that the resources and time involved are compatible with the required game performance. In this question it is sought to identify whether the system responds in an expected way;

- User experience: elements and factors related to interaction with the game.

\section{RESUlTS AND DISCUSSION}

Regarding the software usability, it is notable that it is possible to understand the game operation without the need for major external intervention and have easily readable information, such as the level progression and player score. Overall gameplay showed differences in ratings, where only one user rated the game as easy, while two users rated it as an intermediate difficulty game.
Regarding functionality, users considered that the game meets its needs. Three users said that the game can meet the purpose, but there are still some points to be worked on. It is important to emphasize that the amputee considered the game as a great aid in the process of rehabilitation.

In terms of efficiency, there were important contributions to the evaluation of the game. The system response time to the users' movements was considered fast. This feedback allows us to conclude that the equipment and structure adopted were adequate for the proposal. However, there were times when, although the response was fast, the moves in the virtual environment were not concise with the ones performed in the real environment. Because of this, users felt that the game's efficiency level was not as high as it needed to be.

Users considered that the game presents a high level of difficulty, which is probably a consequence of the way in which the interaction with the objects was made and the reported loss of precision in some moments. In general, users have considered the game to be stimulating, a feature of great importance so that they do not desire to leave the environment during their training

\section{CONCLUSIONS}

There are some points that needs to be adjusted and improved so that the game reaches a more satisfactory level, with a focus on the precision of movements and interaction with the virtual objects.

It was observed that the methods and techniques used for the development of the system were shown to be consistent and adequate for the proposed goal. The amputee user will develop during the process and acquire greater control over the virtual prosthesis, reducing the time needed to adapt to his or her actual prosthesis.

Finally, based on the feedback provided by the research participants, it can be concluded that the serious game developed provides important characteristics for the amputee rehabilitation process.

\section{ACKNOWLEDGMENT}

The authors would like to thank the AutoDesk Foundation, CAPES and FAPEMIG for financial support.

\section{REFERENCES}

[1] Ministerio da Saúde, Diretrizes de atenção a pessoa amputada, 1st ed., M. da Saude, Ed. Brasília: Ministerio da Saúde, 2013.

[2] S. B. PORTER, Fisioterapia em amputados, 13th ed. Rio de Janeiro: Elsevier, 2005.

[3] IBGE, “Censo demografico 2010," https://censo2010.ibge.gov.br, 2010.

[4] S. B. O'sullivan and T. J. Schmitz, "Fisioterapia: avaliação e tratamento," in Fisioterapia: avaliação e tratamento, 2004. 